\title{
Endovascular Revascularization as a Treatment Option for Peripheral Arterial Disease due to Diabetes in Saudi Arabia: Public Awareness Survey
}

\author{
Afnan Hammad Ali Alshammari ${ }^{1}$ Noura Hamad Rasheed Alshurtan ${ }^{1}$ \\ Amirah Fahad Mohammed Alshammeri ${ }^{1}$ Abdulaziz Ayied Abdullah Alharbi ${ }^{2}$
}

${ }^{1}$ College of Medicine, Hail University, Hail, Saudi Arabia
2 Medical Imaging Department, Division of Vascular Interventional
Radiology, King Abdulaziz Medical City National Guard Hospital,
Riyadh, Saudi Arabia
Address for correspondence Afnan Hammad Ali Alshammari, College of Medicine, Hail University, Hail 81411, Saudi Arabia (e-mail: Afnan96h@hotmail.com).

Arab J Intervent Radiol 2021;5:93-96.

\begin{abstract}
Keywords

- diabetes-related peripheral arterial disease

- endovascular revascularization

- interventional radiology

- Saudi Arabia

Objectives Diabetic foot disease is a common and serious complication of diabetes that represents a major global health concern with high mortality and morbidity rates. Successful revascularization and improved perfusion have had fundamental roles in reducing the rate of amputation in diabetic foot patients over the last decade. Creating public awareness plays a key role in early treatment and prevention of amputation. The aim of this study was to determine if people are aware of endovascular revascularization as a therapeutic option for diabetes-related peripheral artery disease.

Methods The study is a community-based observational descriptive cross-sectional study that was conducted to assess Saudi population knowledge and awareness about endovascular revascularization as a treatment option of diabetic foot disease through interventional radiology. The self-administered online survey was randomly distributed with a maximum of 20 multiple choice questions through social media channels.

Results Endovascular revascularization as a treatment of diabetes-related peripheral artery disease through interventional radiology was unknown to $90.2 \%$ of the participants. Awareness has been influenced by many factors including age, occupation, and family history.

Conclusion This study highlights the importance of raising public awareness about endovascular revascularization as a treatment option for diabetes-related peripheral arterial disease in suitable patients.
\end{abstract}

\section{Introduction}

Diabetic foot disease (DFD) is a common and serious complication of diabetes that represents a major health concern with high associated mortality and morbidity rates. DFD is classified under the umbrella of "critical limb ischemia" (CLI), which varies in symptoms from rest pain to ulcers and tissue loss. ${ }^{1}$ DFD is attributed to many causative factors, the most common of which are peripheral sensory published online February 9, 2022
DOI https://doi.org/

$10.1055 / \mathrm{s}-0041-1741113$. ISSN 2542-7075.
(C) 2022. The Pan Arab Interventional Radiology Society. All rights reserved.

This is an open access article published by Thieme under the terms of the Creative Commons Attribution-NonDerivative-NonCommercial-License, permitting copying and reproduction so long as the original work is given appropriate credit. Contents may not be used for commercial purposes, or adapted, remixed, transformed or built upon. (https://creativecommons.org/ licenses/by-nc-nd/4.0/)

Thieme Medical and Scientific Publishers Pvt. Ltd., A-12, 2nd Floor, Sector 2, Noida-201301 UP, India 
neuropathy and peripheral arterial disease (PAD). ${ }^{2}$ In Saudi Arabia, the prevalence of peripheral sensory neuropathy among patients with diabetes is $29.1 \% .^{3}$ PAD has a prevalence rate of approximately $23.1 \%$ in the Saudi diabetic population. ${ }^{4}$ Foot ulcers are associated with an increased risk of lower limb amputation in patients with diabetes. ${ }^{2}$

A study conducted in Saudi Arabia showed that the findings of two previous studies as well as three methodological publications led to the prediction that approximately 325 amputations are likely to occur annually in Jeddah, compared with 741 in Riyadh and 3,970 in Saudi Arabia. ${ }^{5}$ Thus, the main goal of peripheral ischemic foot ulcer treatment is limb preservation. ${ }^{2}$ A multidisciplinary team, including interventional radiologists, vascular surgeons, foot surgeons, podiatrists, endocrinologists, family physicians, and physiotherapists, is essential for the effective treatment of PAD. Successful revascularization and improved perfusion have had fundamental roles in reducing the rate of amputation among diabetic foot patients over the last decade.

Treatment options have been developed for non-healing ischemic DFD, including surgical bypass for the occluded vessels (performed through vascular surgery) or endovascular revascularization (i.e., percutaneous transluminal angioplasty or stenting), which is mostly performed through interventional radiology. Nonsurgical treatment has many advantages. Specifically, effective nonsurgical treatment presents an achievable, safe, minimally invasive, low-risk, and cost-effective set of procedures for limb salvage in a high percentage of diabetic patients. ${ }^{6}$ However, because of the rapid development of new technology, public awareness of the effectiveness of endovascular options in the treatment of diabetic PAD has not been thoroughly investigated, especially in Saudi Arabia. Raising public awareness plays a key role in early treatment and prevention of amputation. This study aimed to assess the level of awareness and knowledge among the Saudi population regarding interventional radiology as an option for the treatment of DFD.

\section{Materials and Methods}

In this cross-sectional study, the self-administered online survey for the year 2021 was distributed at random through social media platforms. A simple random sample of 1,776 participants was calculated using a Raosoft sample size calculator. ${ }^{7}$ The minimum sample size needed to conduct this study with a confidence interval of $99 \%$ was $664 .^{7}$ Data were collected using Google forms (Google Sheets) and were coded and processed using Microsoft Excel and the Software Statistical Package for the Social Science (SPSS) version 23 (SPSS, Inc., Chicago, Illinois, United States).

The survey questionnaire started with an informed consent document, and all participants provided their written informed consent for participating in the study.

The survey questionnaire contained 20 multiple choice questions divided into three main sections (-Supplementary Material S1, available online only). The first section consisted of demographic data, including nationality, region, gender, age, education level, employment in the medical field (students or working professionals), and family history of diabetes ( $\mathbf{- T a b l e ~ 1 ) . ~}$

The second section covered questions about knowledge and perceptions regarding DFD, including definition of DF, current positive family history, associated symptoms, causative factors, DFD treatment options (including conservative treatment with wound care and antibiotics, revascularization, and amputation), and individual opinions regarding DFD as a common complication of diabetes in Saudi Arabia. The third section focused on endovascular revascularization as a treatment option for DFD-related PAD. Questions included familiarity with this procedure, if the procedure was considered minimally invasive, the type of anesthesia used in this intervention, participants' opinions on whether the procedure could contribute to reducing the amputation rate, and the source of their information. The accuracy and validity of the questionnaire were confirmed by two consultants: an interventional radiologist and a family physician.

Descriptive statistics, including frequencies and percentages, were used to describe items and study variables. As these variables represent nominal data, Chi-square tests were conducted to assess differences in variable distribution as well as associations between variables based on the study objectives. Statistical significance was set at a $p$-value $\leq 0.05$.

The study was approved by the Ethical Research Committee of the College of Medicine at the University of Hail, Saudi Arabia. This study was conducted in accordance with the principles of the Declaration of Helsinki.

\section{Result}

A total 1,743 people participated in this study (75.4\% female; $24.6 \%$ male), the majority of them were Saudi (97.4\%). Their age was classified into five groups with the majority of participants in the 41 to 50 and 20 to 30 years age groups (26.4 and 25.8\%, respectively). In addition to that, around two-thirds $(71.8 \%)$ were university students or had Bachelor's degree (-Table $\mathbf{1}$ ).

The survey tool was distributed through social media platforms within the Kingdom of Saudi Arabia and the percentages from each region were as follows: Middle $33.6 \%$, North $27.5 \%$, West $13.2 \%$, East $13.0 \%$, and South region $12.7 \%$

A history of diabetes mellitus was found in $67.5 \%$ of the participants' families. Moreover, $12.1 \%$ of all respondents had a family history of DFD. By using Chi-square test was conducted to evaluate the relationship between the family history of DFD with some demographic parameters. DFD awareness was significantly associated with $34.1 \%$ of the participants $(p=0.001)$ of age group of 41 to 50 years. Similarly, with a central region of SA by $33.6 \%(p=0.000)$.

The reported symptoms of patients with DFD are mentioned in -Table 2. The Table shows that a majority of the patients have poor glycemic control. Regarding the knowledge of DFD, around half of the participants (56.8\%) did not know what DFD is, and even those who knew about it (43.2\%), $8.2 \%$ of the participants had a wrong impression of 
Table 1 Demographic data

\begin{tabular}{|c|c|c|}
\hline & & $\%$ \\
\hline \multirow[t]{2}{*}{ Gender } & Male & 24.6 \\
\hline & Female & 75.4 \\
\hline \multirow[t]{2}{*}{ Nationality } & Saudi & 97.4 \\
\hline & Non-Saudi & 2.6 \\
\hline \multirow[t]{5}{*}{ Age } & Less than 20 & 9.5 \\
\hline & $20-30$ & 25.8 \\
\hline & $31-40$ & 22.3 \\
\hline & $41-50$ & 26.4 \\
\hline & More than 50 & 16 \\
\hline \multirow[t]{4}{*}{ Education level } & Illiterate & 0.5 \\
\hline & $\begin{array}{l}\text { Primary, Middle, or } \\
\text { Secondary school }\end{array}$ & 20.1 \\
\hline & University & 71.8 \\
\hline & Master or PhD & 7.5 \\
\hline
\end{tabular}

Table 2 Diabetic foot disease symptoms

\begin{tabular}{|l|l|}
\hline & $\%$ \\
\hline Poor glycemic control & 30.3 \\
\hline $\begin{array}{l}\text { A feeling of coldness, pain, } \\
\text { burning sensation or tingling in the foot }\end{array}$ & 24.9 \\
\hline Foot redness or hotness & 15.7 \\
\hline Loss of sensory or motor function of the foot & 15.1 \\
\hline Foot deformity & 11.3 \\
\hline
\end{tabular}

it ( - Table 3).We sought to establish relationships associated with the correct definition of DFD and found that the level of education and occupational health were statistically significant. The relation between correct definition of DFD and level of education was characteristically associated with university students by $74.8 \%(p=0.000)$. Likewise, medical students and workers showed an advantage over non-medical students by $86.9 \%(p=0.000)$. Contrarily, the age in relation to knowledge of DFD was statistically insignificant ( $p=0.42$ ).

The highest percentage of people were unaware of the common causes of DFD (54.1\%), given the fact that neuropathy and foot ischemia are the responsible causes, which $42.6 \%$ agree and $3.3 \%$ disagree.

A total of $61.3 \%$ felt that DFD is one of the most common complications of diabetes in Saudi Arabia, 33.9\% had no idea about that and $4.9 \%$ believed that DFD is not considered a common complication.

Awareness of treatment of DFD varied among respondents (-Fig. 1) with the highest two relative percentages between $35.7 \%$ reported that by conservative treatment with wound care and antibiotics and 30.1\% reported that by revascularization. We focused on asking those who had a family history of DFD regarding the treatment options. It appeared that the highest percentage (16.9\%) chose ampu-
Table 3 Public awareness of diabetic foot definition

\begin{tabular}{|l|l|}
\hline Do you know what diabetic foot disease is? & $\%$ \\
\hline Yes & 43.2 \\
\hline No & 56.8 \\
\hline Yes & $43.2 \%$ \\
\hline $\begin{array}{l}\text { An open wound that is difficult to heal } \\
\text { and loss of tissue which is commonly located } \\
\text { on the bottom of the foot }\end{array}$ & 91.8 \\
\hline $\begin{array}{l}\text { Tightening or tearing of the ankle joint } \\
\text { ligaments }\end{array}$ & 2.9 \\
\hline Bony protrusion on the underside of the heel & 2.9 \\
\hline Chronic skin disease or recurrent itching & 2.4 \\
\hline
\end{tabular}

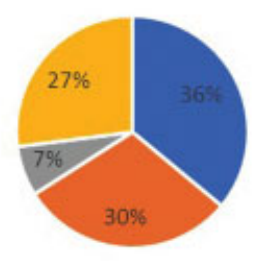

$=$ I do not know

Fig. 1 Public awareness of treatment options of diabetic foot.

tation as a definitive treatment. Around $14.2 \%$ of diabetic patients reported the conservative treatment with wound care and antibiotics as the preferred treatment, $12.8 \%$ selected the revascularization option, and $7.7 \%$ denied any information about the options at all.

As for the awareness about endovascular revascularization for the treatment of DFD, the majority of patients (90.2\%) had never heard about the interventional radiological approach, while only $9.8 \%$ were familiar with it.

Notably, the knowledge and awareness of endovascular revascularization have been influenced by many factors such as age, occupation, and family history. The median age group 20 to 30 years is well aware of the procedure by $30.4 \%$. Almost equal percentage between 41 to 50 years (22.6\%) and 31 to 40 years (20.0\%). It was also a statistically significant outcome in relation to employment/training in the medical field (90.5\%) and in family history of DFD (87.9\%).

Concerns about the interventional radiology approach, to be considered as safe and minimally invasive, were investigated among the familiar group. It showed that $62.5 \%$ had no concerns, $8.3 \%$ had some worries, and $29.2 \%$ were unsure whether it is a risky procedure or not.

A total of $56.3 \%$ of the participants were aware about local anesthesia, which is the type of anesthesia used to perform this procedure, while $30.5 \%$ did not know about it, and $13.2 \%$ reported it as a general anesthesia. The fact that the endovascular revascularization can contribute to the reduction of the amputation rate of the diabetic foot was approved by 
$73.8 \%$ of the participants, $19 \%$ had no idea about it, and $7.1 \%$ did not agree with it.

We found that the largest source of the participants' information were social media $28.7 \%$ and internet $26.2 \%$ followed by friends or relatives $16.0 \%$, family physician $10.2 \%$, medical books $10.2 \%$, and the patient's surgeon $8.6 \%$.

\section{Discussion}

Endovascular intervention allows for a minimally invasive low-risk procedure and it can be performed under local anesthesia, and thus public health efforts are necessary to promote knowledge of this treatment modality within the Saudi population. ${ }^{8}$

In two studies conducted in Saudi Arabia, patients had no knowledge of interventional radiology procedures as treatment choices for their medical conditions. According to a study conducted in $2020,74.2 \%$ of participants had never heard of radiofrequency ablation as a thyroid nodule treatment option. ${ }^{9}$ Another study of Saudi women's awareness of uterine artery embolization found that $76.1 \%$ had never heard of this procedure. ${ }^{10}$ This current study is the first to evaluate the Saudi population's awareness of interventional radiology procedures for the treatment of DFD. Majority of respondents in this online survey had no knowledge of interventional radiology treatment options for DFD. Involvement in the medical field, whether by employment or study, had a major impact on awareness of interventional radiology treatment options for DFD. These deficiencies arise from a lack of awareness about treatment options, whereas public health education and specialist consultation prevent amputation.

\section{Conclusion}

The findings of this study highlight the need for further efforts to raise public awareness of the importance of the role of endovascular intervention in the treatment of DFD with poor perfusion, as important medical decisions require patients to be well-informed and active in the decisionmaking process.

\section{Financial Disclosures \\ None.}

\section{Ethics Approval}

This study was approved by the Research Ethics Committee of the University of Hail (approval number: 42-538862). All participants provided their written informed consent. This study was conducted in accordance with the principles of the Declaration of Helsinki.

\section{Authors' Contributions}

A.H.A.A. and N.H.R.A. contributed toward study design, literature search, questionnaire design, data collection, data analysis, data interpretation, manuscript preparation, manuscript editing, manuscript review, and approval of the current version of the manuscript. A.F.M.A. did the manuscript preparation, manuscript editing, manuscript review, and approval of the current version of the manuscript. A.A. A.A. contributed toward study design, literature search, questionnaire design, data interpretation, manuscript preparation, manuscript editing, and manuscript review.

\section{Conflict of Interest}

None declared.

\section{Acknowledgments}

The authors would like to express their gratitude to all participants in the study.

\section{References}

1 Dua A, Lee CJ. Epidemiology of peripheral arterial disease and critical limb ischemia. Tech Vasc Interv Radiol 2016;19(02): 91-95

2 Elgzyri T, Larsson J, Nyberg P, Thörne J, Eriksson KF, Apelqvist J. Early revascularization after admittance to a diabetic foot center affects the healing probability of ischemic foot ulcer in patients with diabetes. Eur J Vasc Endovasc Surg 2014;48(04):440-446

3 Sendi RA, Mahrus AM, Saeed RM, Mohammed MA, Al-Dubai SAR. Diabetic peripheral neuropathy among Saudi diabetic patients: a multicenter cross-sectional study at primary health care setting. J Family Med Prim Care 2020;9(01):197-201

4 Guraya SY, London NJM. The prevalence and management strategies for peripheral artery disease associated with diabetes mellitus in the Arab world. J Taibah Univ Med Sci 2016;11(04):310-316

5 Alzahrani HA. Diabetes-related lower extremities amputations in Saudi Arabia: the magnitude of the problem. Ann Vasc Dis 2012;5 (02):151-156

6 Kolte D, Kennedy KF, Shishehbor MH, et al. Endovascular versus surgical revascularization for acute limb ischemia: a propensityscore matched analysis. Circ Cardiovasc Interv 2020;13(01):e008150

7 Raosoft. Sample Size Calculator. Accessed January 1, 2021 at: http://www.raosoft.com/samplesize.html

8 Reekers JA. Interventional radiology in the diabetic lower extremity. Med Clin North Am 2013;97(05):835-845

9 Albaqawi R, Alreshidi M, Almuhelb A, Aladel F. Awareness, knowledge and attitudes regarding thyroid nodules in Saudi Arabia. 2020

10 Alreshidi MN, Alshubrmi D, Alrashidi I, Arabi M. Awareness of uterine artery embolization as a treatment for fibroids among Saudi Women. Arab J Interv Radiol 2020;4(02):79-82

11 Sharma G, Farber A, Menard MT. Endovascular or Open Surgical Therapy for Critical Limb Ischemia. American College of Cardiology; 2017

12 Wiseman JT, Fernandes-Taylor S, Saha S, et al. Endovascular vs. open revascularization for peripheral arterial disease. Ann Surg 2017;265(02):424-430 